Research paper

\title{
Neuroanatomical predictors of L-DOPA response in older adults with psychomotor slowing and depression: A pilot study
}

\author{
Bret R Rutherford ${ }^{\mathrm{a}, *}$, Jongwoo Choi ${ }^{\mathrm{a}}$, Mark Slifstein ${ }^{\mathrm{b}}$, Kaleigh O'Boyle ${ }^{\mathrm{a}}$, Anissa Abi-Dargham ${ }^{\mathrm{b}}$, \\ Patrick J Brown ${ }^{\mathrm{a}}$, Melanie W Wall ${ }^{\mathrm{a}}$, Nora Vanegas-Arroyave ${ }^{c}$, Jayant Sakhardande ${ }^{c}$, \\ Yaakov Stern ${ }^{c}$, Steven P Roose ${ }^{a}$ \\ ${ }^{a}$ Columbia University College of Physicians and Surgeons, New York State Psychiatric Institute, New York, NY, United States \\ ${ }^{\mathrm{b}}$ Stony Brook University College of Medicine, New York, NY, United States \\ ${ }^{\mathrm{c}}$ Columbia University College of Physicians and Surgeons, New York, NY, United States
}

\section{A R T I C L E I N F O}

\section{Keywords:}

Late life depression

Processing speed

Gait speed

Levodopa

Magnetic resonance imaging

\begin{abstract}
A B S T R A C T
Background: Declining function in dopamine circuits is implicated in normal aging and late-life depression (LLD). Dopamine augmentation recently has shown therapeutic promise, but predictors of response are unknown.

Methods: Depressed elders with slowed gait underwent baseline magnetic resonance imaging (MRI) and [ $\left.{ }^{11} \mathrm{C}\right]$ raclopride positron emission tomography (PET). Subjects then received open treatment with carbidopa/levodopa (L-DOPA) for three weeks. Linear regressions examined relationships between baseline MRI measures, $\left[{ }^{11} \mathrm{C}\right]$ raclopride binding, and behavioral outcomes.

Results: Among $N=16$ participants aged $72.5 \pm 6.8$ years, higher left superior temporal gyrus volume was associated with higher processing speed at baseline, while cortical thinning in a processing speed network was associated with greater improvement following L-DOPA. Greater volume and cortical thickness in brain regions associated with mobility were associated with higher baseline gait speed. Higher baseline white matter hyperintensity volume predicted less post-L-DOPA improvement on dual task gait speed and IDS-SR scores. Higher $\left[{ }^{11} \mathrm{C}\right]$ raclopride binding in the associative striatum was associated with cortical thickness in some, but not all, processing speed brain regions, while higher binding in sensorimotor striatum was significantly associated with left caudate volume.

Limitations: Limiting the conclusions drawn from this pilot study are the small sample size and open administration of L-DOPA.

Conclusions: Greater baseline brain volumes and cortical thickness in regions supporting cognition and gait were associated with higher behavioral performance, while lower structural integrity was associated with increased responsivity to L-DOPA. If substantiated in larger studies, these findings could facilitate the targeting of dopaminergic treatments to those LLD patients most likely to respond.
\end{abstract}

\section{Introduction}

Late life depression (LLD) is a prevalent and disabling condition among older adults that is often recurrent, can become chronic, and is frequently non-responsive to antidepressant medication (Consensus Conference, 1992; Rothschild, 1996; Alexopoulos et al., 1996; Sneed et al., 2008). Executive Dysfunction (ED), particularly as manifested in slowed processing speed, is common in patients with LLD, predicts poorer acute response to antidepressants, and is associated with higher relapse rates during the continuation phase (Butters et al.,
2004; Pimontel et al., 2012). Similarly, slowed gait speed is associated with incident depression in older adults and interacts with depression to magnify adverse health outcomes (e.g., falls, loss of independent living status, mortality) in older adults (Demakakos et al., 2013; Brown et al., 2016; Verghese et al., 2009; Van Kan et al., 2009). Recently, our group targeted slowed processing/gait speed and decreased striatal dopamine availability, which is an important physiologic basis for slowing, using a novel therapeutic approach to LLD. We showed that carbidopa/levodopa (L-DOPA) monotherapy significantly improved processing and gait speed in depressed older adults, decreased $\left[{ }^{11} \mathrm{C}\right]$

\footnotetext{
* Corresponding author.

E-mail address: brr8@cumc.columbia.edu (B.R. Rutherford).
} 
raclopride binding in selected striatal subregions, and resulted in decreased depressive symptoms (Rutherford et al., 2019).

Given that L-DOPA administration is a novel therapeutic strategy for LLD with cognitive and motor slowing, little is known about brain regions that may be associated with response. L-DOPA is most frequently employed for the treatment of Parkinson's disease (PD), which typically is not associated with identifiable changes on traditional structural brain imaging with T1- and T2-weighted sequences (Stoessl, 2011). In addition, the hypodopaminergic state associated with aging has a distinct neurobiology from PD and appears to represent a parallel pathway to developing cognitive and motor deficits in LLD (Bohnen et al., 2006; Volkow et al., 1998), so it may be unlikely that predictors of L-DOPA response are shared across LLD and PD. In the only study available examining structural MRI variables and therapeutic response to LDOPA for PD, greater brain atrophy in the parietal operculum, primary and supplementary motor areas, and cerebellum were associated with relatively weak L-DOPA response (Ballarini et al., 2019).

Predictors of response to standard antidepressant medication treatment for LLD include white matter hyperintensities (WMH) observed on T2-weighted fluid attenuated inversion recovery (FLAIR) MRI scans (Sneed et al., 2011). WMH are present in the majority of older adults with MDD and are associated with a more chronic clinical course and poor response to antidepressants (Sheline et al., 2010; Aizenstein et al., 2014). Gray matter structural alterations also have been linked to the development and maintenance of LLD, and studies integrating neuroimaging with antidepressant treatment have reported smaller anterior cingulate (Gunning et al., 2009) and hippocampal volumes (Sheline et al., 2012) are associated with decreased antidepressant response. However, given the different mechanisms of therapeutic action for dopaminergic agents and standard antidepressants, it is unclear whether these findings are relevant to L-DOPA response.

In this secondary analysis of our previously reported L-DOPA study, the primary goal was to examine associations between structural MRI variables and L-DOPA response on processing speed, gait speed, and depressive symptom outcomes. Our guiding hypotheses were that 1) higher brain volume and cortical thickness in regions underlying processing and gait speed would be associated with better behavioral performance on corresponding measures at baseline, whereas 2) lower baseline brain volume and cortical thickness would be associated with increased response to L-DOPA on the corresponding behavioral tasks at study endpoint. Moreover, we wished to characterize associations between WMH burden and psychomotor speed at baseline as well as evaluate whether, similar to what has been found with respect to treatment with antidepressant medication, greater baseline WMH burden is associated with decreased responsivity to L-DOPA on the three outcomes studied (i.e., processing speed, gait speed, and depressive symptoms). Finally, to explore possible pathophysiologic pathways linking age-associated declines in dopamine signaling with cortical thinning, regional atrophy, and psychomotor slowing, we examined associations between $\left[{ }^{11} \mathrm{C}\right]$ raclopride binding and baseline structural MRI measures in brain regions linked to processing and gait speed.

\section{Methods and materials}

\subsection{Subjects}

This study was conducted in the Adult and Late Life Depression Research Clinic at the New York State Psychiatric Institute (NYSPI) and approved by the NYSPI Institutional Review Board. Please see the previously published main outcome manuscript for a full description of study procedures (Rutherford et al., 2019). The analyses reported here concern the subset of our original study's participants who underwent MRI scanning, as structural MRI data were not presented previously. Briefly, eligible subjects were adult outpatients aged $\geq 60$ years who were diagnosed with Diagnostic and Statistical Manual (DSM) 5 MDD,
Dysthymia, or Depression Not Otherwise Specified (NOS), had Center for Epidemiologic Studies-Depression Rating scale (CES-D) score $\geq 10$, and decreased gait speed (average walking speed over $15^{\prime}$ course < $1 \mathrm{~m} / \mathrm{s}$ ). Subjects were excluded for substance abuse or dependence, psychosis or bipolar disorder, diagnosis of probable dementia, Mini Mental Status Examination (MMSE) $\leq 24$, HRSD suicide item $>2$ or Clinical Global Impressions (CGI)-Severity score of 7 at baseline, within the past 4 weeks treatment with psychotropic or other medications known to affect dopamine, acute or severe medical illness, mobility limiting osteoarthritis or joint disease, or contraindication to magnetic resonance imaging (MRI).

\subsection{Study design and L-DOPA administration}

Neuroimaging participants had pre-treatment MRI and PET scans prior to a Week 0 visit, after which they began taking $37.5 \mathrm{mg}$ carbidopa/150 mg levodopa once daily (9am). After one week at this dosage, subjects were instructed to take $37.5 \mathrm{mg}$ carbidopa/150 mg levodopa at 9am and $5 \mathrm{pm}$ (twice daily). For the third week of treatment, subjects took $37.5 \mathrm{mg}$ carbidopa/150 mg levodopa three times daily (9am, $12 \mathrm{pm}, 5 \mathrm{pm}$ ). Participants were instructed to maintain the same timing of doses throughout the study as described below and continued for the three-week duration study.

\subsection{Study assessments}

Processing and gait speed were assessed at baseline, Week 0 , and weekly upon initiation of L-DOPA treatment (i.e., Weeks 1-3). Assessments were performed at approximately $1 \mathrm{pm}$ to control for time of day effects and the duration since the last morning L-DOPA dose (anticipated to be $4 \mathrm{~h}$ ). Processing speed was assessed using the Digit Symbol test from the Wechsler Adult Intelligence Scale-III (WAIS-III) (Wechsler, 1997) and the Pattern and Letter Comparison tests (Salthouse and Babcock, 1991). A latent factor constructed from these three measures was chosen as the primary outcome measure for processing speed, with a higher score indicating improved processing speed. Gait speed was measured in $\mathrm{m} / \mathrm{s}$ both as a single task in which study participants walked at their usual or normal speed on a 15 foot walking course and as a dual task in which participants walked at their usual speed while naming as many animals as possible. Two trials (each, for single and dual tasks) were completed, and the final gait speed measurement was recorded as the average of these two trials.

Other study measurements performed weekly included the 24-item HRSD, the Inventory of Depressive Symptomatology 30-item self-report (IDS-SR), the CGI-Severity and CGI-Improvement scales, a rating scale for treatment-emergent side effects, and weekly pill counts.

\subsection{MRI data acquisition and analysis}

MRI of the brain was acquired using a GE MR750 3.0T System, beginning with a 3-Plane localizer (scout) to determine patient position. Next, a T1-weighted (BRAVO) scan was performed with TI $450 \mathrm{~ms}$, flip angle of $12^{\circ}$, in-plane resolution of $256 \times 256$, field of view of $25.6 \times 25.6 \mathrm{~cm}$, and 180 slices in oblique plane with slice-thickness/ gap of $1.0 \mathrm{~mm}$. Finally, T2 FLAIR for WMH quantification was acquired with TE $120 \mathrm{~ms}$, TR $10000 \mathrm{~ms}$, TI $2380 \mathrm{~ms}$, flip angle of $111^{\circ}$, field of view of $22.0 \times 22.0 \mathrm{~cm}$, and 50 slices in oblique plane with slicethickness/gap of $3.0 \mathrm{~mm}$. FreeSurfer (v5.1.0) software for human brain imaging analysis (http://surfer.nmr.mgh.harvard.edu/) was used for the reconstruction of the T1 scans (Fischl et al., 2002; Fischl et al., 2004). Regional brain volumes and thickness in cortical regions was computed using the standard FreeSurfer parcellation (Desikan et al., 2006).

For associations with baseline processing speed and L-DOPA-associated change on this measure, we selected for analysis volume (mean right and left, $\mathrm{mm}^{3}$ ) and thickness (mean right and left, $\mathrm{mm}$ ) for brain 
Table 1

Baseline and week 3 clinical and demographic measures for included subjects.

\begin{tabular}{|c|c|c|c|c|c|}
\hline Characteristic & Baseline (mean $\pm \mathrm{SD}$ ) & Week 3 (mean \pm SD) & $\mathrm{t}$ & df & $\mathrm{p}$ \\
\hline Age & $72.5 \pm 6.8$ & & & & \\
\hline$\%$ male & 56.3 & & & & \\
\hline Education (yrs) & $15.6 \pm 2.6$ & & & & \\
\hline MMSE & $27.5 \pm 1.6$ & & & & \\
\hline Processing speed factor & $-3.3 \pm 6.7$ & $0.4 \pm 5.0$ & 3.574 & 15 & 0.003 \\
\hline Digit Symbol Test & $30.9 \pm 10.0$ & $40.6 \pm 7.9$ & 4.706 & 15 & $<0.001$ \\
\hline Pattern comparison test & $11.8 \pm 2.8$ & $12.9 \pm 1.9$ & 2.358 & 15 & 0.033 \\
\hline Letter comparison test & $6.5 \pm 2.4$ & $6.6 \pm 2.0$ & 0.809 & 14 & 0.43 \\
\hline Single task gait speed & $0.77 \pm 0.19$ & $0.89 \pm 0.22$ & 3.230 & 15 & 0.006 \\
\hline Dual task gait speed & $0.61 \pm 0.21$ & $0.78 \pm 0.24$ & 3.656 & 15 & 0.002 \\
\hline 24-item HRSD & $15.9 \pm 5.8$ & $10.6 \pm 7.4$ & -2.747 & 15 & 0.015 \\
\hline IDS-SR & $25.9 \pm 10.9$ & $13.4 \pm 9.6$ & -6.434 & 15 & $<0.001$ \\
\hline CGI Severity & $3.3 \pm 0.8$ & $2.5 \pm 1.1$ & -2.467 & 14 & 0.027 \\
\hline
\end{tabular}

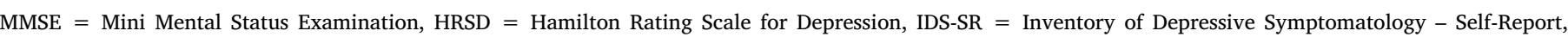
CGI $=$ Clinical Global Impressions.

regions that we have previously shown to be activated during performance of the processing speed tasks used in this study (Habeck et al., 2015). These comprise lingual gyrus, thalamus, insular cortex, cuneus, precuneus, and superior/middle temporal cortex. For gait speed analyses, we selected brain regions that have been associated with gait speed in previous reports (Yuan et al., 2015): caudate, putamen, pallidum, hippocampus, primary motor cortex, and supplementary motor area. Finally, total WMH burden was selected for comparison with baseline and change measures as described below.

\subsection{Positron emission tomography scanning}

Dynamically acquired $\left[{ }^{11} \mathrm{C}\right]$ raclopride scans were performed on a Biograph mCT hybrid PET/CT scanner (Siemens, Knoxville, TN). Initially, a brief $(<10 \mathrm{~s})$ CT scan of the head was acquired for attenuation correction. Following intravenous injection of $\left[{ }^{11} \mathrm{C}\right]$ raclopride, emission data were collected in list mode for $60 \mathrm{~min}$. List mode data were binned into a sequence of frames of increasing duration (from $20 \mathrm{~s}$ to $10 \mathrm{~min}$ ) and reconstructed by filtered backprojection, with correction for attenuation, random coincidences, scatter and dead time, using manufacturer-provided software. High resolution anatomical T1weighted MRI scans were acquired for each subject and PET data were coregistered to the MRIs using maximization of mutual information (SPM12, Wellcome Centre for Human Neuroimaging). Regions of interest (ROIs) were applied to the MRIs and transferred to the PET emission data and included the sensorimotor striatum (post-commissural putamen, SMST) and associative striatum (whole caudate and precommissural putamen, AST) as previously described (Martinez et al., 2004). Additionally, an ROI was drawn on cerebellum as a reference tissue. ROI time activity curves were derived as the average activity in each ROI in each frame.

\subsection{Statistical analyses}

Means were computed for clinical and demographic characteristics of the study sample, and a latent factor analysis was conducted to create a processing speed factor from digit symbol, pattern comparison, and letter comparison test scores. The relationships between structural brain measures (i.e., volume, thickness) and outcomes of interest were examined using linear regressions. Separate regressions modeled each baseline outcome measure (i.e., processing speed factor, single or dual task gait speed, total depressive symptoms as measured by 24-item HRSD, $\left[{ }^{11} \mathrm{C}\right]$ raclopride binding in striatal ROIs) as a function of each brain region of interest (volume and thickness). We hypothesized that structural brain measures would be associated with baseline behavioral and PET measures in a regionally specific fashion, such that volume/ thickness in the six processing speed regions would be associated with baseline processing speed factor scores and volume/thickness in the six gait regions would be associated with baseline gait speed. Moreover, we expected that volume/thickness in the six processing speed regions would be associated with $\left[{ }^{11} \mathrm{C}\right]$ raclopride binding in AST and volume/ thickness in the six gait regions would be associated with $\left[{ }^{11} \mathrm{C}\right]$ raclopride binding in SMST. Control variables in all regressions were age at scan, sex, educational attainment, and intra-cranial volume. The same brain regions were evaluated in regression models of change, but the outcome was Week3 - Week 0 change in each behavioral/symptom measure and baseline values of the behavioral measure were added as a control. We opted not to utilize a Bonferonni correction for multiple comparisons, as given the small sample size in this pilot study, this type of correction would mask nearly all findings.

\section{Results}

\subsection{Sample characteristics and response to L-DOPA}

Clinical and demographic characteristics for the $N=16$ subjects undergoing MRI scanning are provided in Table 1 . Of these, $N=14$ also had evaluable baseline $\left[{ }^{11} \mathrm{C}\right]$ raclopride data. These participants represented the MRI-eligible portion of the previously published sample who received treatment with L-DOPA (Rutherford et al., 2019). Scanned participants had a mean age of $72.5 \pm 6.8$ years, were $56.3 \%$ male, and had mean HRSD $15.9 \pm 5.8$. Mean baseline processing speed as measured by the Digit Symbol test was $30.9 \pm 10.0$, which is $>1$ SD below norms for individuals having similar age, sex, and education characteristics (Gaertner et al., 2018). Mean single task gait speed was $0.77 \pm 0.19$, and mean dual task gait speed was $0.61 \pm 0.21$, both of which are $>1$ SD below the age-adjusted norm for age 74 (closest published norm to the mean age of the current sample) (Beauchet et al., 2017).

Among the $N=16$ neuroimaging dataset, L-DOPA treatment was associated with improvements in processing (processing speed factor $t=3.574$, df $15, p=0.003$ ) and gait speed (single task: $t=3.230$, df $15, p=0.006$; dual task: $t=3.656$, df $15, p=0.002$ ) from Week 0 to Week 3 . In addition, statistically significant improvements in depressive symptoms was observed from Week 0 to Week 3 on the HRSD $(t=-2.747, \mathrm{df} 15, p=0.015)$ and IDS-SR $(t=-6.434$, df 15 , $p<0.001)$.

\subsubsection{Relationships between structural brain measures and baseline psychomotor speed}

Among brain regions previously shown to underlie performance on tests of processing speed, greater left superior temporal gyrus volume ( $t$ \#x003D; 3.119, df 9, p \#x003D; 0.012) was significantly associated with better processing speed factor scores at baseline (see Table 2). As 
Table 2

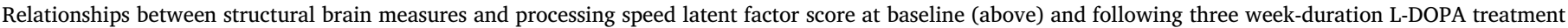
(below).

\begin{tabular}{|c|c|c|c|c|c|c|}
\hline $\begin{array}{l}\text { Baseline } \\
\text { Brain region }\end{array}$ & $\begin{array}{l}\text { Volume } \\
\mathrm{t}\end{array}$ & df (residual) & $\mathrm{p}$ & $\begin{array}{l}\text { Thickness } \\
\mathrm{t}\end{array}$ & df (residual) & $\mathrm{p}$ \\
\hline $\begin{array}{l}\text { superior temporal gyrus LR } \\
\text { Change following L-DOPA }\end{array}$ & 3.1190 .855 & 99 & 0.0120 .415 & 0.8420 .160 & 99 & 0.4220 .877 \\
\hline Brain region & $\begin{array}{l}\text { Volume } \\
\mathrm{t}\end{array}$ & df (residual) & $\mathbf{p}$ & $\begin{array}{l}\text { Thickness } \\
\text { t }\end{array}$ & df (residual) & $\mathbf{p}$ \\
\hline Cuneus LR & -1.7490 .629 & 88 & 0.1180 .547 & $-2.281-2.745$ & 88 & 0.0520 .025 \\
\hline Insula L R & 0.2840 .229 & 88 & 0.7840 .825 & $-1.369-2.457$ & 88 & 0.2080 .039 \\
\hline lingual gyrus $L \mathrm{R}$ & $-0.233-0.847$ & 88 & 0.8210 .421 & $-3.194-3.991$ & 88 & 0.0130 .004 \\
\hline Precuneus L R & $-0.397-2.061$ & 88 & 0.7020 .073 & $-1.500-2.966$ & 88 & 0.1720 .018 \\
\hline superior temporal gyrus $\mathrm{L} R$ & 0.7950 .777 & 88 & 0.4500 .459 & $-2.953-0.888$ & 88 & 0.0180 .400 \\
\hline
\end{tabular}

Table 3

Relationships between structural brain measures and gait speed at baseline (above) and following three week-duration L-DOPA treatment (below).

\begin{tabular}{|c|c|c|c|c|c|c|}
\hline $\begin{array}{l}\text { Baseline } \\
\text { Brain volumes }\end{array}$ & $\begin{array}{l}\text { Single Task Gait Speed } \\
\mathrm{t}\end{array}$ & df (residual) & $\mathrm{p}$ & $\begin{array}{l}\text { Dual Task Gait Speed } \\
\mathrm{t}\end{array}$ & df (residual) & $\mathrm{p}$ \\
\hline $\begin{array}{l}\text { supplementary motor cortex LR } \\
\text { Change following L-DOPA }\end{array}$ & 2.9211 .882 & 99 & 0.0170 .092 & 2.6410 .989 & 99 & 0.0270 .349 \\
\hline \multirow[t]{2}{*}{ Brain volumes } & \multicolumn{3}{|l|}{ Single Task Gait Speed } & \multicolumn{3}{|l|}{ Dual Task Gait Speed } \\
\hline & $\mathrm{t}$ & df (total) & $\mathbf{p}$ & $\mathrm{t}$ & df (total) & $\mathbf{p}$ \\
\hline \multicolumn{7}{|l|}{ Baseline } \\
\hline Brain thickness & $\begin{array}{l}\text { Single Task Gait Speed } \\
\mathrm{t}\end{array}$ & $\begin{array}{l}\text { Dual Task Gait Speed } \\
\text { df (total) }\end{array}$ & $\mathbf{p}$ & $\mathbf{t}$ & df (total) & $\mathbf{P}$ \\
\hline primary motor cortex (anterior) LR & 2.112 .75 & 99 & 0.0640 .022 & 1.571 .66 & 99 & 0.1510 .132 \\
\hline $\begin{array}{l}\text { supplementary motor cortex LR } \\
\text { Change following L-DOPA }\end{array}$ & 1.432 .31 & 99 & 0.1860 .046 & 1.272 .23 & 99 & 0.2360 .052 \\
\hline Brain thickness & Single Task Gait Speed & & & Dual Task Gait Speed & & \\
\hline & t & df (total) & $\mathbf{p}$ & $\mathrm{t}$ & df (total) & $\mathbf{p}$ \\
\hline supplementary motor cortex L R & $-0.749-2.122$ & 88 & 0.4750 .067 & $-0.699-0.646$ & 88 & 0.5040 .537 \\
\hline
\end{tabular}

shown in Table 3, higher left supplementary motor cortex volume was associated with faster baseline single task $(t \#$ x003D; 2.921, df 9, $p$ \#x003D; 0.017) and dual task (2.641, df 9, $p=0.027)$ gait speed. Right primary motor $(t=2.75$, df $9, p=0.022)$ and supplementary motor area $(t=2.31$, df $9, p=0.046)$ cortical thickness also were associated with higher single task gait speed. No significant associations between volumetric measures and baseline processing or gait speed were found in the remaining regions examined. Significance testing for these comparisons was not corrected for multiple comparisons given the small sample size in this pilot study.

\subsubsection{Associations between structural MRI variables and L-DOPA effects} on processing and gait speed

As shown in Table 2, lower right cuneus $(t=-2.745$, df 8 , $p=0.025)$, right insula $(t=-2.457$, df $8, p=0.039)$, bilateral lingual gyrus (left: $t=-3.194$, df $8, p=0.013$; right: $t=-3.991$, df 8 , $p=0.004)$, right precuneus $(t=-2.966$, df $8, p=0.018)$, and left superior temporal gyrus $(t=-2.953$, df $8, p$ \#x003D; 0.018) cortical thickness at baseline were associated with greater improvement on the processing speed factor score following L-DOPA treatment. Higher left caudate volume ( $t=2.493$, df $8, p=0.037)$ was found to be associated with greater post-L-DOPA improvement on the dual task gait speed measure.

Total WMH burden was not associated with any baseline measures. Higher baseline WMH volumes were associated with less post-L-DOPA improvement on dual task gait speed $(t=-5.164$, df $3, p=0.014)$ and IDS-SR scores $(t=3.664$, df $3,0.035)$ but not with change in processing speed. As in the above section, significance testing for these comparisons was not corrected for multiple comparisons given the small sample size in this pilot study.

\subsubsection{Associations between $\left[{ }^{11} C\right]$ raclopride binding and structural MRI} measures

Higher baseline $\left[{ }^{11} \mathrm{C}\right]$ raclopride binding in AST was significantly associated with cortical thickness in some, but not all, processing speed brain regions, including bilateral insular (left: $t=4.120$, $\mathrm{df} 7$, $p=0.004$; right: $t=2.693$, df $7, p=0.31$ ) and left superior temporal gyrus ( $t=3.346$, df $7, p=0.012)$. With respect to associations between PET measures and gait speed brain regions, higher baseline $\left[{ }^{11} \mathrm{C}\right]$ raclopride binding in SMST was significantly associated with left caudate volume ( $t=5.081$, df $7, p=0.001$ ). Significance testing for these comparisons was not corrected for multiple comparisons given the small sample size in this pilot study.

\section{Discussion}

In these analyses of structural MRI data, $\left[{ }^{11} \mathrm{C}\right]$ raclopride binding, and psychomotor speed in depressed older adults, lower regional brain volume and cortical thickness in areas previously linked to processing speed were associated with poorer baseline performance on processing speed tasks and increased cognitive responsivity to L-DOPA monotherapy. Similarly, lower integrity of neural structures subserving motor functions was linked to slower gait speed at baseline. WMH burden, a well-described moderator of depressive symptom response to antidepressant medication in older adults, was associated with decreased dual task gait speed and depressive symptom responses to LDOPA but was not associated with processing speed improvements in this study.

By non-invasively providing information about the integrity of brain circuits underlying dopamine-dependent behaviors, structural MRI data such as those analyzed here could facilitate the development and targeted application of personalized interventions for LLD. Previous 
studies have examined patient-level moderators of response to antidepressant medications such as selective serotonin reuptake inhibitors (Gunning et al., 2009; Sheline et al., 2012), but these findings may not be relevant to therapeutic strategies targeting alternative mechanisms in subgroups of depressed older adults. Results reported here suggest that it is older adults with atrophy in brain regions underlying information processing speed who may benefit most from dopaminergic interventions to increase processing speed. Given the 'inverted Ushaped' function believed to characterize the relationship between dopamine signaling and performance across motor and cognitive domains (Arnsten, 1998; Cools, 2006), it may be desirable to target dopaminergic interventions to individuals with a signaling deficit. Though not specifically examined here, using L-DOPA in individuals with high baseline dopamine levels and intact frontostriatal pathways may result in 'over-dosing' and a decline in performance.

We also found that baseline $\left[{ }^{11} \mathrm{C}\right]$ raclopride binding in the AST was significantly associated with structural MRI measures in the processing speed network examined, while $\left[{ }^{11} \mathrm{C}\right]$ raclopride binding in the SMST was significantly associated with left caudate volume. While interpretation of baseline $\left[{ }^{11} \mathrm{C}\right]$ raclopride binding values in these striatal subregions is complex, the findings reported here are consistent with a model in which age-related declines in dopaminergic input to the striatum result in downstream atrophy among frontal cortex regions subserving cognitive (anterior cingulate cortex, ventrolateral and dorsolateral PFC) and motor (primary motor cortex and supplemental motor area) functions. Further study is needed to more conclusively examine the relationships between age-related change in dopaminergic inputs (i.e., from the ventral tegmental area and substantia nigra) and neuroplastic changes in downstream cortical and subcortical structures. A related question for future studies is whether L-DOPA treatment alters volume and cortical thickness in brain regions subserving processing and gait speed, on what time scale such changes occur, and whether they persist following removal of L-DOPA treatment.

Consistent with the bulk of previous research on antidepressant treatment response in LLD, higher WMH burden was associated with poorer treatment outcomes. Interestingly, this obtained both for motor (dual task gait speed) and depressive symptom outcomes, suggesting that cerebrovascular lesions may be an important negative prognostic factor across different pharmacologic treatments and diverse outcome domains. Along with targeted pharmacologic and psychotherapeutic treatment for depression, a comprehensive therapeutic strategy for LLD in elders with risk factors or evidence of WMH should involve vigorous management of cardiovascular health.

These results must be interpreted with caution given the small sample size available for analysis, which is the primary limitation of the findings reported here. The limited sample size did not permit controlling for multiple comparisons, so it is possible that one or more of the findings reported here may represent a Type I error. Moreover, LDOPA was administered as an open treatment in this study, and expectancy-based placebo effects may have contributed to the brain-behavior associations identified.

Mitigating these limitations are the fact that, to our knowledge, no prior studies have examined associations between brain structure and response to dopaminergic treatments for LLD. Given that dopamine circuits are implicated in the pathogenesis of depression across the lifespan and that aging is associated with functionally significant dopaminergic decline, it likely that dopaminergic interventions may come to play important roles in the treatment of LLD. The findings reported here may best be viewed as valuable hypothesis-generating pilot data to be further evaluated by future, adequately powered studies. Such a study is currently underway in our laboratories, where $N=90$ depressed older adults are being randomized to treatment with L-DOPA $450 \mathrm{mg}$ or placebo in addition to undergoing pre- and post-treatment multimodal MRI and PET imaging.

Future studies examining dopaminergic circuits in LLD also may benefit from integrating additional MRI sequences with the volumetric approach utilized in this study. For example, diffusion tensor imaging to examine the integrity of specific tracts linking PFC subregions to subcortical structures that are important for mood regulation (e.g., superior longitudinal fasciculus, uncinate fasciculus, anterior thalamic radiations) may be a more sensitive method for capturing white matter abnormalities in LLD than overall WMH volume (Wen et al., 2014). Additionally, recent studies validating neuromelanin-sensitive MRI (NMMRI) as an in vivo proxy of dopaminergic function suggest that this modality may be particularly useful for measuring dopaminergic dysfunction in LLD (Cassidy et al., 2019). Neuromelanin is a product of dopamine synthesis that accumulates in midbrain nuclei over the lifespan, particularly in the substantia nigra pars compacta (SNc), but decreases through degeneration of dopamine neurons (as in PD). Future studies may examine the extent to which older depressed patients have reduced SNc neuromelanin and whether this is associated with responsivity to L-DOPA and other dopaminergic treatments.

\section{Author disclosure}

This work was supported by the National Institutes of Health (NIMH R61 MH110029; Principal Investigator Rutherford).

Drs. Rutherford, Brown, Roose, Stern, Vanegas-Arroyave, Wall, Ms. O'Boyle, and Mr. Choi and Sakhardande have no financial disclosures or conflicts of interest to report. Dr. Abi-Dargham reports being a stockholder in Systems 1 Bio and Storm Biosciences, receiving honoraria for lectures from Otsuka, and being a member of scientific advisory boards with Roche and Sunovion. Dr. Slifstein reports being a stockholder in Systems 1 Bio and Storm Biosciences.

This study was approved by the New York State Psychiatric Institute Institutional Review Board as Protocol \#7270 and registered on Clinicaltrials.gov (NCT02744391).

\section{Author statement}

The work described in this manuscript has not been published previously, nor is it under consideration for publication elsewhere. The primary clinical and PET findings of the project described were published previously as cited in the manuscript (i.e., Rutherford BR, Slifstein M, Chen C, Abi-Dargham A, Brown PJ, Wall MW, VanegasArroyave N, Stern Y, Bailey V, Valente E, Roose SP. Effects of L-DOPA Monotherapy on Psychomotor Speed and $\left[{ }^{11} \mathrm{C}\right]$ Raclopride Binding in High Risk Depressed Older Adults. Biol Psychiatry 2019; 86:221-229.).

\section{Contributors}

All authors of this manuscript have materially participated in the work described and have had the opportunity to approve the final article. Specific roles of each author were as follows: Rutherford-study design, data collection, data analysis, manuscript preparation; Roose, Abi-Dargham, Stern-study design, manuscript preparation; O'Boyle, Vanegas-Arroyave-data collection; Choi, Slifstein, Wall-data analysis. Our disclosures can be found in the main manuscript file.

\section{Role of the funding source}

This work was supported by the National Institutes of Health (NIMH R61 MH110029; Principal Investigator Rutherford). As corresponding author, I have had access to all of the data reported in this manuscript and have had complete freedom to direct its reporting.

\section{Acknowledgements}

There are no acknowledgements for this manuscript. 


\section{References}

Consensus Conference, N.I.H., 1992. Diagnosis and treatment of depression in late life JAMA 268, 1018-1024.

Rothschild, A.J., 1996. The diagnosis and treatment of late-life depression. J. Clin. Psychiatry 57, 5-11.

Alexopoulos, G.S., Meyers, B.S., Young, R.C., Kakuma, T., Feder, M., Einhorn, A., Rosedahl, E., 1996. Recovery in geriatric depression. Arch. Gen. Psychiatry 53, 305-312.

Sneed, J.R., Rutherford, B.R., Rindskopf, D., Roose, S.P., 2008. Design makes a difference: antidepressant response rates in placebo-controlled versus comparator trials in late life depression. Am. J. Geri. Psychiatry 16, 65-73.

Butters, M.A., Whyte, E., Nebes, R.D., et al., 2004. The nature and determinants of neuropsychological functioning in late-life depression. Arch. Gen. Psychiatry 61, 587-595.

Pimontel, M.A., Culang-Reinlieb, M.E., Morimoto, S.S., Sneed, J.R., 2012. Executive dysfunction and treatment response in late life depression. Int. J. Geriatr. Psychiatry 27, 893-899.

Demakakos, P., Cooper, R., Hamer, M., de Oliveira, C., Hardy, R., Breeze, E., 2013. The bidirectional association between depressive symptoms and gait speed: evidence from the English Longitudinal Study of Aging (ELSA). PLoS ONE 8https://doi.org/10. 1371/journal. pone.0068632. e68632.

Brown, P.J., Roose, S.P., Zhang, J., et al., 2016. Inflammation, depression, and slow gait: a high mortality phenotype in later life. J. Gerontol. A Biol. Sci. Med. Sci. 71, 221-227.

Verghese, J., Holtzer, R., Lipton, R.B., Wang, C., 2009. Quantitative gait markers and incident fall risk in older adults. J. Gerontol. A Biol. Sci. Med. Sci. 64, 896-901.

Van Kan, A.G., Rolland, Y., Andrieu, S., et al., 2009. Gait speed at usual pace as a predictor of adverse outcomes in community-dwelling older people: an international academy on nutrition and aging (IANA) task force. J. Nutr. Health Aging 13, 881-889.

Rutherford, B.R., Slifstein, M., Chen, C., Abi-Dargham, A., Brown, P.J., Wall, M.W., Vanegas-Arroyave, N., Stern, Y., Bailey, V., Valente, E., Roose, S.P., 2019. Effects of LDOPA monotherapy on psychomotor speed and [11C]Raclopride binding in high risk depressed older adults. Biol. Psychiatry 86, 221-229.

Stoessl, A.J., 2011. Neuroimaging in parkinson's disease. Neurotherapeutics 8, 72-81.

Bohnen, N.I., Albin, R.L., Koeppe, R.A., Wernette, K.A., Kilbourn, M.R., Minoshima, S., Frey, K.A., 2006. Positron emission tomography of monoaminergic vesicular binding in aging and parkinson disease. J. Cereb. Blood Flow Metab. 26, 1198-1212.

Volkow, N.D., Wang, G.J., Fowler, J.S., et al., 1998. Parallel loss of presynaptic and postsynaptic dopamine markers in normal aging. Ann. Neurol. 44, 143-147.

Ballarini, T., Mueeler, K., Albrecht, F., et al., 2019. Regional gray matter changes and age predict individual treatment response in parkinson's disease. NeuroImage Clin 101636.

Sneed, J.R., Culang-Reinlieb, M.E., Brickman, A.M., et al., 2011. MRI signal hyperintensities and failure to remit following antidepressant treatment. J. Affect. Disord. 135, 315-320.

Sheline, Y.I., Pieper, C.F., Barch, D.M., et al., 2010. Support for the vascular depression hypothesis in late-life depression. Arch. Gen. Psychiatry 67, 277-286.
Aizenstein, H.J., Khalef, A., Walker, S.E., Andreescu, C., 2014. MRI predictors of treatment response in late-life depression. J. Geriatr. Psychiatry Neurol. 27, 24-32.

Gunning, F.M., Cheng, J., Murphy, C.F., Kanellopoulos, D., Acuna, J., Hoptman, M.J., Klimstra, S., Morimoto, S., Weinberg, J., Alexopoulos, G.S., 2009. Anterior cingulate cortical volumes and treatment remission of geriatric depression. Int. J. Geriatr. Psychiatry 24, 829-836.

Sheline Y.I., Disabato B.M., Hranilovich J., et al. Treatment course with antidepressant therapy in late-life depressionAm. J. Psychiatry 2012; 169:1185-1193.

Wechsler, D., 1997. Wechsler memory scale - 3rd ed. San Antonio, TX. Psychol. Corp.

Salthouse, T.A., Babcock, R.L., 1991. Decomposing adult age differences in working memory. Dev. Psychol. 27, 763-776.

Fischl, B., Salat, D.H., Busa, E., et al., 2002. Whole brain segmentation: automated labeling of neuroanatomical structures in the human brain. Neuron 33, 341-355.

Fischl, B., Salat, D.H., van der Kouwe, A.J., et al., 2004. Sequence-independent segmentation of magnetic resonance images. Neuroimage 23 (Suppl 1), S69-S84.

Desikan, R.S., Segonne, F., Fischl, B., et al., 2006. An automated labeling system for subdividing the human cerebral cortex on MRI scans into gyral based regions of interest. Neuroimage 31, 968-980.

Habeck, C., Steffener, J., Barulli, D., Gazes, Y., Shaked, D., Salthouse, T., Stern, Y., 2015. Making cognitive latent variables manifest: distinct neural networks for fluid reasoning and processing speed. J. Cogn. Neurosci. 27, 1249-1258.

Yuan, J., Blumen, H.M., Verghese, J., Holtzer, R., 2015. Functional connectivity associated with gait velocity during walking and walking-while-talking in aging: a resting state fMRI study. Hum. Brain Mapp. 36, 1484-1493.

Martinez, D., Broft, A., Foltin, R.W., Slifstein, M., Hwang, D.R., Huang, Y., et al., 2004. Cocaine dependence and D-2 receptor availability in functional subdivisions of the striatum: relationship with cocaine-seeking behavior. Neuropsychopharmacology 29, 1190-1202.

Gaertner, B., Wagner, M., Luck, T., Buttery, A.K., Fuchs, J., Busch, M.A., 2018. Normative data for the digit symbol substitution test in a population-based sample aged 65 to 79 years: results from the German Health Interview and Examination Survey for Adults (DEGS1). Clin. Neuropsychol. https://doi.org/10.1080/13854046.2018.

Beauchet, O., Allali, G., Sekhon, H., Verghese, J., Guilain, S., Steinmetz, J.P., et al., 2017. Guidelines for assessment of gait and reference values for spatiotemporal gait parameters in older adults: the biomathics and Canadian gait consortiums initiative. Front. Hum. Neurosci. 11, 353.

Arnsten, A.F.T., 1998. Catecholamine modulation of prefrontal cortical cognitive function. Trends Cogn. Sci. 2, 436-446.

Cools, R., 2006. Dopaminergic modulation of cognitive function-implications for L-DOPA treatment in parkinson's disease. Neurosci. Biobehav. Rev. 30, 1-23.

Wen, M.C., Steffens, D.C., Chen, M.K., Zainal, N.H., 2014. Diffusion tensor imaging studies in late-life depression: systematic review and meta-analysis. Int. J. Geriatr. Psychiatry 29, 1173-1184.

Cassidy, C.M., Zucca, F.A., Girgis, R.R., Baker, S.C., Weinstein, J.J., Sharp, M.E., Bellei, C., Valmadre, A., Vanegas, N., Kegeles, L.S., Brucato, G., Jung Kang, U., Sulzer, D., Zecca, L., Abi-Dargham, A., Horga, G., 2019. Neuromelanin-sensitive MRI as a noninvasive proxy measure of dopamine function in the human brain. Proc. Natl. Acad. Sci. U. S. A. $116,5108-5117$. 